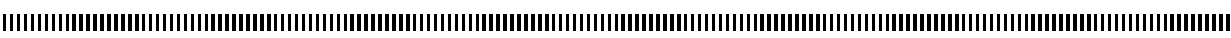

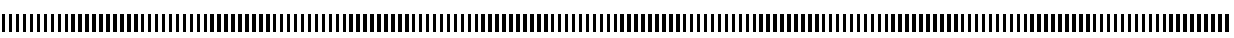
|

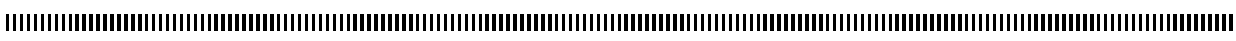
| |

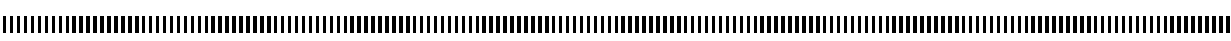

CARI'10

\title{
Collision-resistant hash function based on composition of functions
}

\author{
René Ndoundam ${ }^{a, b}$, Juvet Karnel Sadié ${ }^{a, b}$ \\ ${ }^{a}$ Université de Yaoundé I, UMI 209, UMMISCO, B.P. 337 Yaoundé, Cameroun \\ ${ }^{b}$ Université de Yaoundé I, LIRIMA, Equipe GRIMCAPE, Faculté des Sciences, Département d'In- \\ formatique, B.P. 812 Yaoundé, Cameroun \\ E.mail : ndoundam@gmail.com,ndoundam@yahoo.com, karnel12@yahoo.fr
}

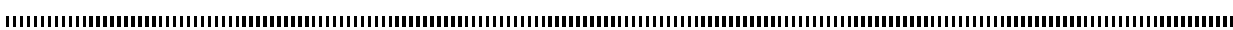

RÉSUMÉ. Une fonction de hachage cryptographique est une procédure déterministe qui compresse un ensemble de données numériques de taille arbitraire en une chaîne de bits de taille fixe. Il existe plusieurs fonctions de hachage : MD5, HAVAL, SHA... II a été reporté que ces fonctions de hachage ne sont pas sécurisées. Notre travail a consisté à la construction d'une nouvelle fonction de hachage basée sur une composition de fonctions. Cette construction utilise la NP-completude des tables de contingence de dimension 3 et une relaxation de la contrainte selon laquelle une fonction de hachage doit être aussi une fonction de compression.

ABSTRACT. A cryptographic hash function is a deterministic procedure that compresses an arbitrary block of numerical data and returns a fixed-size bit string. There exists many hash functions: MD5, HAVAL, SHA, ... It was reported that these hash functions are no longer secure. Our work is focused on the construction of a new hash function based on composition of functions. The construction used the NP-completeness of Three-dimensional contingency tables and the relaxation of the constraint that a hash function should also be a compression function.

MOTS-CLÉS : NP-complet, fonction à un sens, Matrice des zéros et des uns, table de contingence de dimension 3 , fonction de hachage résistante aux collisions.

KEYWORDS : NP-complete, One-way function, Matrix of zeros and ones, Three-dimensional contingency table, Collision-resistant hash function.

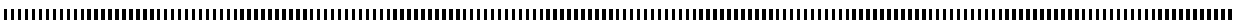




\section{Introduction}

A cryptographic hash function is a deterministic procedure that compresses an arbitrary block of data and returns fixed-size bit string, the hash value (message digest or digest). An accidental or intentional change of the data will almost certainly change the hash value. Hash functions are used to verify the integrity of data or data signature.

Let us suppose that $h: X \rightarrow Y$ is a hash function without key. The function $h$ is secured if the following three problems are difficult to solve.

Problem 1 : First Preimage attack

Instance : a function $h: X \rightarrow Y$ and an image $y \in Y$

Query: $\quad x \in X$ such that $h(x)=y$

We suppose that a possible hash $y$ is given, we want to know if there exists $x$ such that $h(x)=y$. If we can solve First Preimage attack, then $(x, y)$ is a valid pair. A hash function for which First Preimage attack can't be solved efficiently is sometimes called Preimage resistant.

Problem 2 : Second Preimage attack

Instance : a function $h: X \rightarrow Y$ and an element $x_{1} \in X$

Query: $\quad x_{2} \in X$ such that $x_{1} \neq x_{2}$ and $h\left(x_{1}\right)=h\left(x_{2}\right)$

A message $x_{1}$ is given, we want to find a message $x_{2}$ such that $x_{2} \neq x_{1}$ and $h\left(x_{1}\right)=$ $h\left(x_{2}\right)$. If this is possible, then $\left(x_{2}, h\left(x_{1}\right)\right)$ is a valid pair. A function for which Second preimage attack can't be solve efficiently is sometimes called Second preimage resistant.

Problem 3 : Collision attack

Instance : a function $h: X \rightarrow Y$

Query: $\quad x_{1}, x_{2} \in X$ such that $x_{1} \neq x_{2}$ and $h\left(x_{1}\right)=h\left(x_{2}\right)$

We want to known if it is possible to find two distinct messages $x_{1}$ and $x_{2}$ such that $h\left(x_{1}\right)=h\left(x_{2}\right)$. A function for which Collision attack can't be solve efficiently is sometimes called Collision resistant.

There exists many hash functions : MD4, MD5, SHA-0, SHA-1, RIPEMD, HAVAL. It was reported that such widely hash functions are no longer secured $[7,8,10,11,12$, $13,14]$. Thus, new hash functions should be studied. The existing hash functions such as MD4, MD5, SHA-0, SHA-1, RIPEMD, HAVAL... want to achieve two goals at the same time :

$a^{\circ}$ ) For any input $x$, they return a hash of $x$ (of fixed length, this length depends on the hash function choosed)

$\left.b^{\circ}\right)$ Preimage resistant, Second Preimage resistant and Collision Resistant.

Our contribution is to separate the two goals defined in points $a^{\circ}$ ) and $b^{\circ}$ ). Our hash function $H_{3}$ is defined as follows :

$-\mathrm{H}_{3}=\mathrm{H}_{2} \mathrm{oH}$, 
- $\mathrm{H}_{2}$ is a classical hash function such as MD5, SHA-0, SHA-1, RIPEMD, HAVAL, ....

- Given a $y$, find $x$ such that $H_{1}(x)=y$ is NP-Complete,

- Find $x_{1}, x_{2}$ such that $x_{1} \neq x_{2}$ and $H_{1}\left(x_{1}\right)=H_{1}\left(x_{2}\right)$ is NP-Complete,

- For any input $x$, the length of $H_{1}(x)$ is not fixed. This is the main difference with the classical hash functions.

The paper is organized as follows : in Section 2, some preliminaries are presented. Section 3 is devoted to the design of our hash function. Concluding remarks are stated in Section 4.

\section{Preliminaries}

Let's define some preliminaries useful for the next section.

\subsection{Two-dimensional}

Data security in two dimension have been studied by many authors $[2,3,9,17]$. Let $m$ and $n$ be two positive integers, and let $R=\left(r_{1}, r_{2}, \ldots, r_{m}\right)$ and $S=\left(s_{1}, s_{2}, \ldots, s_{n}\right)$ be non-negative integral vectors. Denoted by $\mathfrak{A}(R, S)$ the set of all $m \times n$ matrices $A=\left(a_{i j}\right)$ satisfying

$$
\begin{gathered}
a_{i j}=0 \text { or } 1 \text { for } i=1,2, \ldots, m \text { and } j=1,2, \ldots, n ; \\
\sum_{j=1}^{n} a_{i j}=r_{i} \text { for } i=1,2, \ldots, m ; \\
\sum_{i=1}^{m} a_{i j}=s_{j} \text { for } j=1,2, \ldots, n .
\end{gathered}
$$

Thus a matrix of 0 's and 1's belongs to $\mathfrak{A}(R, S)$ provided its row sum vector is $R$ and its column sum vector is $S$. The set $\mathfrak{A}(R, S)$ was studied by many authors $[1,4,5,6,16]$. Ryser [16] has defined an interchange to be a transformation which replaces the $2 \times 2$ submatrix :

$$
B_{0}=\left(\begin{array}{ll}
1 & 0 \\
0 & 1
\end{array}\right)
$$

of a matrix A of 0 's and 1 's with the $2 \times 2$ submatrix

$$
B_{1}=\left(\begin{array}{ll}
0 & 1 \\
1 & 0
\end{array}\right)
$$

If the submatrix $B_{0}$ (or $B_{1}$ ) lies in rows $k, l$ and columns $u, v$, then we call the interchange a $(k, l ; u, v)$-interchange. An interchange (or any finite sequence of interchanges) does not alter the row and column sum vectors of a matrix. Ryser has shown the following result.

Theorem 1 [16] Let $A$ and $A^{*}$ be two $m$ and $n$ matrices composed of 0 's and l's, possessing equal row sum vectors and equal column sum vectors. Then $A$ is transformable into $A^{*}$ by a finite number of interchanges. 
Let us consider a matrix $A \in\{0,1\}^{n \times n} \in \mathfrak{A}(R, S)$, i.e. its row sum vector $R$ is such that $R \in\{0,1,2, \ldots, n\}^{n}$ and its column sum vector $S$ is such that $S \in\{0,1,2, \ldots, n\}^{n}$. We define the function $g_{1}$ from $\{0,1\}^{n \times n}$ to $\mathbb{N}^{2 n}$ as follows :

$$
\begin{aligned}
g_{1}(A)= & R(1)\|R(2)\| \ldots\|R(n)\| \\
& S(1)\|S(2)\| \ldots \| S(n)
\end{aligned}
$$

where $\|$ denotes the concatenation.

\subsection{Three-dimensional}

Irving and Jerrum [15] have studied the extension of the problem in three dimension and shown that problems that are solvable in polynomial time in the two-dimensional case become NP-Complete. Suppose that for a given $n \times n \times n$ table $D$ of non-negative integers, and for each $i, j, k$, the row, column and file sums are denoted by $R(i, k), C(j, k)$ and $F(i, j)$ respectively. In other words :

$$
\begin{aligned}
& R(i, k)=\sum_{j=1}^{n} D(i, j, k) \\
& C(j, k)=\sum_{i=1}^{n} D(i, j, k) \\
& F(i, j)=\sum_{k=1}^{n} D(i, j, k)
\end{aligned}
$$

The following problem is studied by Irving and Jerrum [15] :

Problem 4. Three-dimensional contingency tables (3DCT)

Instance : A positive integer $n$, and for each $i, j, k$ non-negative integers values $R(i, k), C(j, k)$ and $F(i, j)$

Question : Does there exist an $n \times n \times n$ contingency table $X$ of non-negative integers such that :

$$
\begin{aligned}
& \sum_{j=1}^{n} X(i, j, k)=R(i, k) \\
& \sum_{i=1}^{n} X(i, j, k)=C(j, k) \\
& \sum_{k=1}^{n} X(i, j, k)=F(i, j)
\end{aligned}
$$

for all $i, j, k$ ? Irving and Jerrum show the following result :

Corollary 1 [15] 3DCT is NP-Complete, even in the special case where all the row, column and file sums are 0 or 1 . 
Let us consider a matrix $A \in \mathbb{N}^{n \times n \times n}$ such that its row sum matrix is a matrix $R$ such that $R \in \mathbb{N}^{n \times n}$ (i.e. $R(i, k) \in \mathbb{N}$ ), the column sum matrix is a matrix $C$ such that $C \in \mathbb{N}^{n \times n}$ (i.e. $C(j, k) \in \mathbb{N}$ ) and the file sum matrix is a matrix $F$ such that $F \in \mathbb{N}^{n \times n}$ (i.e. $F(i, j) \in \mathbb{N}$ ). We define the function $g_{2}$ as follows :

$$
\begin{aligned}
& g_{2}: \mathbb{N}^{n \times n \times n} \longrightarrow \mathbb{N}^{3 n^{2}} \\
g_{2}(A)= & R(1,1)\|R(1,2)\| \ldots\|R(n, n)\| \\
& C(1,1)\|C(1,2)\| \ldots\|C(n, n)\| \\
& F(1,1)\|F(1,2)\| \ldots \| F(n, n)
\end{aligned}
$$

Let us consider the following matrices $A$ and $B$. We define the element product of matrices $A$ and $B$ as follows :

Definition 1 Element Product of Matrices of dimension 2

Let $A, B \in \mathbb{R}^{n_{1} \times n_{2}}$, we define the Element product of matrices $A$ and $B$ as follows :

$C=A * B$; where $c_{i j}=a_{i j} \times b_{i j}$ for $\mathrm{i}, \mathrm{j}$ such that

$$
1 \leq i \leq n_{1} \text { and } 1 \leq j \leq n_{2}
$$

Definition 2 Element Product of Matrices of dimension 3

Let $A, B \in \mathbb{R}^{n_{1} \times n_{2} \times n_{3}}$, we define the Element product of matrices $A$ and $B$ as follows :

$C=A . * B ;$ where $c_{i j k}=a_{i j k} \times b_{i j k}$ for $\mathrm{i}, \mathrm{j}, \mathrm{k}$ such that

$$
1 \leq i \leq n_{1}, 1 \leq j \leq n_{2} \text { and } 1 \leq k \leq n_{3}
$$

\section{Design of the hash function}

Before the construction of our hash function, let us explain the main idea.

\subsection{Explanation of the idea by an example}

In page 175 of paper [1], Brualdi gives the example of the following five matrices :

$$
\begin{gathered}
A_{1}=\left(\begin{array}{lll}
1 & 1 & 0 \\
1 & 1 & 0 \\
0 & 0 & 1
\end{array}\right) ; A_{2}=\left(\begin{array}{lll}
1 & 1 & 0 \\
1 & 0 & 1 \\
0 & 1 & 0
\end{array}\right) ; A_{3}=\left(\begin{array}{lll}
1 & 1 & 0 \\
0 & 1 & 1 \\
1 & 0 & 0
\end{array}\right) \\
A_{4}=\left(\begin{array}{lll}
0 & 1 & 1 \\
1 & 1 & 0 \\
1 & 0 & 0
\end{array}\right) ; A_{5}=\left(\begin{array}{lll}
1 & 0 & 1 \\
1 & 1 & 0 \\
0 & 1 & 0
\end{array}\right)
\end{gathered}
$$

which belong to $\mathfrak{A}(R, S)$ where $R=S=(2,2,1)$. Let us note $W$ the following matrix :

$$
W=\left(\begin{array}{ccc}
1 & 4 & 9 \\
2 & 8 & 18 \\
3 & 12 & 27
\end{array}\right)
$$


Based on the Element Product of Matrix defined in the previous subsection, it is easy to verify that :

$$
\begin{gathered}
A_{1} * W=\left(\begin{array}{ccc}
1 & 4 & 0 \\
2 & 8 & 0 \\
0 & 0 & 27
\end{array}\right) ; A_{2} * W=\left(\begin{array}{ccc}
1 & 4 & 0 \\
2 & 0 & 18 \\
0 & 12 & 0
\end{array}\right) ; A_{3} * W=\left(\begin{array}{ccc}
1 & 4 & 0 \\
0 & 8 & 18 \\
3 & 0 & 0
\end{array}\right) \\
A_{4} \cdot * W=\left(\begin{array}{ccc}
0 & 4 & 9 \\
2 & 8 & 0 \\
3 & 0 & 0
\end{array}\right) ; A_{5} \cdot * W=\left(\begin{array}{ccc}
1 & 0 & 9 \\
2 & 8 & 0 \\
0 & 12 & 0
\end{array}\right)
\end{gathered}
$$

By computation, we evaluate that :

$$
\begin{aligned}
& g_{1}\left(A_{1} * W\right)=5|| 10|| 27|| 3|| 12|| 27 \quad g_{1}\left(A_{2} * W\right)=5|| 20|| 12|| 3|| 16|| 18 \\
& g_{1}\left(A_{3} * W\right)=5|| 26|| 3|| 4|| 12\left\|18 \quad g_{1}\left(A_{4} * W W\right)=13\right\| 10|| 3|| 5|| 12|| 9 \\
& g_{1}\left(A_{5} \cdot * W\right)=10\|10\| 12|| 3\|\mid 20\| 9
\end{aligned}
$$

It is easy to verify that $A_{1}(2,2) \neq A_{2}(2,2), A_{1}(2,3) \neq A_{2}(2,3), A_{1}(3,2) \neq$ $A_{2}(3,2)$ and $A_{1}(3,3) \neq A_{2}(3,3)$. All these differences imply that

- the second term of $g_{1}\left(A_{1} * W\right)$ is not equal to the second term of $g_{1}\left(A_{2} \cdot * W\right)$,

- the third term of $g_{1}\left(A_{1} * W\right)$ is not equal to the third term of $g_{1}\left(A_{2} * W\right)$,

- the fifth term of $g_{1}\left(A_{1} * W\right)$ is not equal to the fifth term of $g_{1}\left(A_{2} * W\right)$,

- the sixth term of $g_{1}\left(A_{1} * W\right)$ is not equal to the sixth term of $g_{1}\left(A_{2} * W\right)$.

More formally, from the construction of $g_{1}$, we can deduce easily that if $A(i, j) \neq$ $B(i, j)$, then :

$\left.c^{\circ}\right)$ the i-th term of $g_{1}(A * W)$ would probably be different from the $\mathrm{i}$-th term of $g_{1}(B . * W)$,

$\left.d^{\circ}\right)$ the $(\mathrm{n}+\mathrm{j})$-th term of $g_{1}(A * W)$ would probably be different from the $(\mathrm{n}+\mathrm{j})$-th term of $g_{1}(B . * W)$.

From the fact that $3 D C T$ which is related to $g_{2}$ (this is an extension of $g_{1}$ ) is NPComplete, we deduce that :

$\left.e^{\circ}\right)$ Given $y$ and a matrix $W$, find a matrix $A$ such that $g_{2}(A * W)=y$ is NP-Complete.

Our idea is to build a new hash function $\mathrm{H}_{3}$ such that $\mathrm{H}_{3}=\mathrm{H}_{2} \mathrm{OH}$ where

- $H_{2}$ is a classical hash function such as MD5, SHA-0, SHA-1, RIPEMD, HAVAL,...

$-H_{1}$ is a function which exploits the ideas presented in $\left.c^{\circ}\right), d^{\circ}$ ) and $e^{\circ}$ ).

Let us denote $\operatorname{VOnes}(n)$ the vector such that $\operatorname{VOnes}(n) \in\{0,1\}^{n}$ and each of its elements is equal to 1 . Also, let us denote $\operatorname{MOnes}(n)$ the matrix such that $\operatorname{MOnes}(n) \in$ $\{0,1\}^{n \times n}$ and each of its elements is equal to 1 . in other words :

$$
\begin{aligned}
& \operatorname{VOnes}(n)_{i}=1 \text { where } 1 \leq i \leq n \\
& \operatorname{Mones}(n)_{i, j}=1 \text { where } 1 \leq i, j \leq n
\end{aligned}
$$

We denote $N_{+}$the set of strictly positive natural number defined as follows :

$$
N_{+}=\mathbb{N} \backslash\{0\}=\{1,2,3,4, \ldots\}
$$


In the next sub-section, we formalize the observation made in points $c^{\circ}$ ) and $d^{\circ}$ ) and we take into account the NP-Completeness of 3DCT to build a new hash function.

\subsection{Construction of the new hash function}

For any integers $a$ and $p$ such that $0 \leq a \leq-1+2^{p}$, let us denote $\operatorname{bin}(a, p)$ the decomposition of the integer $a$ in base 2 on $p$ positions. In other words :

$$
\operatorname{bin}(a, p)=x_{p-1} x_{p-2} \ldots x_{1} x_{0} \text { and } \sum_{i=0}^{p-1} x_{i} \times 2^{i}=a
$$

Let us also define the following function :

$$
f_{0}(n)=\left\lceil\log _{2}(n+1)\right\rceil
$$

$f_{0}(n)$ represents the number of bits necessary to represent any integer between 0 and $n$ in base 2 .

We also define the following functions :

$$
\begin{aligned}
& f_{1}(A)=\max \left\{\sum_{j=1}^{n} \sum_{k=1}^{n} A(i, j, k): 1 \leq i \leq n\right\}, \\
& f_{2}(A)=\max \left\{\sum_{i=1}^{n} \sum_{k=1}^{n} A(i, j, k): 1 \leq j \leq n\right\}, \\
& f_{3}(A)=\max \left\{\sum_{i=1}^{n} \sum_{j=1}^{n} A(i, j, k): 1 \leq k \leq n\right\}, \\
& f_{4}(A)=\max \left\{f_{1}(A), f_{2}(A), f_{3}(A)\right\} .
\end{aligned}
$$

$f_{4}(A)$ represents the maximun of sum of any $n$ consecutive elements of the matrix $A$ belonging to the same row, or to the same column or to the same file. $f_{0} o f_{4}(A)$ represents the number of bits necessary to represent in base 2 the sum of any $n$ consecutive elements of the matrix $A$ belonging to the same row, or to the same column, or to the same file.

Subsequently, in the aim to be more precise, we redefine $g_{2}$ as follows :

$$
\begin{aligned}
g_{2}(A)= & \operatorname{bin}\left(R(1,1), f_{0} \circ f_{4}(A)\right)\left\|\operatorname{bin}\left(R(1,2), f_{0} \circ f_{4}(A)\right)\right\| \ldots\left\|\operatorname{bin}\left(R(n, n), f_{0} \circ f_{4}(A)\right)\right\| \\
& \operatorname{bin}\left(C(1,1), f_{0} \circ f_{4}(A)\right)\left\|\operatorname{bin}\left(C(1,2), f_{0} \circ f_{4}(A)\right)\right\| \ldots\left\|\operatorname{bin}\left(C(n, n), f_{0} \circ f_{4}(A)\right)\right\| \\
& \operatorname{bin}\left(F(1,1), f_{o} \circ f_{4}(A)\right)\left\|\operatorname{bin}\left(F(1,2), f_{0} \circ f_{4}(A)\right)\right\| \ldots \| \operatorname{bin}\left(F(n, n), f_{0} \circ f_{4}(A)\right)
\end{aligned}
$$

Let us define the following problem :

\section{Problem 5 :}

Instance : A positive integer $n$, two binary strings $x$ and $y$ 
two matrices $V, W \in \mathbb{N}^{n \times n \times n}$

Query : Find two matrices $A, B \in\{0,1\}^{n \times n \times n}$

such that:

$$
\begin{gathered}
A \neq B \\
g_{2}(A * * V)=g_{2}(B * * V)=x \\
g_{2}(A . * W)=g_{2}(B * * W)=y
\end{gathered}
$$

Let us characterize the complexity of Problem5.

Proposition 1 Problem 5 is NP-Complete.

\section{Proof Idea of Proposition 1 :}

We want to show how to transform a solution of 3DCT to a solution of Problem 5 . Without loss of generality, we work in dimension 2. Let us suppose that we want to find a matrix $A \in\{0,1\}^{3 \times 3}$ such that :

$$
\begin{aligned}
& \sum_{j=1}^{3} A(i, j)=R(i) \\
& \sum_{i=1}^{3} A(i, j)=C(j)
\end{aligned}
$$

where $R=(3,2,1)$ and $C=(2,3,1)$.

It is easy to see that the determination of the matrix $A \in\{0,1\}^{3 \times 3}$ which verifies Equations (1) is also equivalent to determining the matrix $B \in\{0,1\}^{6 \times 6}$ such that :

$$
\begin{aligned}
& \sum_{j=1}^{6} B(i, j)=R d(i) \\
& \sum_{i=1}^{6} B(i, j)=C d(j)
\end{aligned}
$$

where $R d=(3,2,1,3,2,1)$ and $C d=(2,3,1,2,3,1)$.

Remark 1 : $R d$ ( respectively $C d$ ) is a duplication of $R$ ( respectively $C$ ).

It is easy to see that from the matrix :

$$
A=\left(\begin{array}{lll}
1 & 1 & 1 \\
1 & 1 & 0 \\
0 & 1 & 0
\end{array}\right)
$$

which verifies Equations (1), we can associate the two following matrices $B_{2}$ and $B_{3}$

$$
B_{2}=\left(\begin{array}{cc}
A & 0_{3 \times 3} \\
0_{3 \times 3} & A
\end{array}\right)=\left(\begin{array}{cccccc}
1 & 1 & 1 & 0 & 0 & 0 \\
1 & 1 & 0 & 0 & 0 & 0 \\
0 & 1 & 0 & 0 & 0 & 0 \\
0 & 0 & 0 & 1 & 1 & 1 \\
0 & 0 & 0 & 1 & 1 & 0 \\
0 & 0 & 0 & 0 & 1 & 0
\end{array}\right)
$$




$$
B_{3}=\left(\begin{array}{cc}
0_{3 \times 3} & A \\
A & 0_{3 \times 3}
\end{array}\right)=\left(\begin{array}{cccccc}
0 & 0 & 0 & 1 & 1 & 1 \\
0 & 0 & 0 & 1 & 1 & 0 \\
0 & 0 & 0 & 0 & 1 & 0 \\
1 & 1 & 1 & 0 & 0 & 0 \\
1 & 1 & 0 & 0 & 0 & 0 \\
0 & 1 & 0 & 0 & 0 & 0
\end{array}\right)
$$

which verify Equations (2). This is the idea of the transformation which associates to one solution of the problem defined in Equations (1) two distinct solutions of the problem defined in Equations (2).

Before the proof, let us introduce the function duplic (which is pseudo-duplication ) of $\mathrm{x}$. We note :

$$
x=x(1) x(2) \ldots x(p)
$$

where $x(i) \in\{0,1\}$ and $p=3 \times n^{2} \times\left\lceil\log _{2}(n+1)\right\rceil$. We define the function $t$ as follows :

$$
t(i, n)=i \times n \times\left\lceil\log _{2}(n+1)\right\rceil ; 0 \leq i \leq 3 n .
$$

The function duplic is defined as follows :

$$
\begin{aligned}
\operatorname{duplic}(x, n)= & d \operatorname{copy}(x, 1, n)\|\operatorname{dcopy}(x, 2, n)\| \ldots\|d \operatorname{copy}(x, n, n)\| \\
& d \operatorname{copy}(x, 1, n)\|d \operatorname{copy}(x, 2, n)\| \ldots\|d \operatorname{copy}(x, n, n)\| \\
& d \operatorname{copy}(x, n+1, n)\|d \operatorname{copy}(x, n+2, n)\| \ldots\|d \operatorname{copy}(x, 2 n, n)\| \\
& d \operatorname{copy}(x, n+1, n)\|d \operatorname{copy}(x, n+2, n)\| \ldots\|d \operatorname{copy}(x, 2 n, n)\| \\
& d \operatorname{dcopy}(x, 2 n+1, n)\|d \operatorname{dcopy}(x, 2 n+2, n)\| \ldots\|d \operatorname{copy}(x, 3 n, n)\| \\
& d \operatorname{copy}(x, 2 n+1, n)\|d \operatorname{dcopy}(x, 2 n+2, n)\| \ldots \| d \operatorname{copy}(x, 3 n, n)
\end{aligned}
$$

where $d \operatorname{copy}(x, i, n)$ is defined as follows :

$$
\operatorname{dcopy}(x, i, n)=\operatorname{strcopy}(x, i, n) \| \operatorname{strcopy}(x, i, n)
$$

and

$$
\operatorname{strcopy}(x, i, n)=x(1+t(i-1, n)) x(2+t(i-1, n)) \ldots x(t(i, n))
$$

For illustration, duplic $(x, 3)$ is defined as follows :

$$
\begin{aligned}
\text { duplic }(x, 3)= & x(1) \ldots x(6) x(1) \ldots x(6) x(7) \ldots x(12) x(7) \ldots x(12) x(13) \ldots x(18) x(13) \ldots x(18) \| \\
& x(1) \ldots x(6) x(1) \ldots x(6) x(7) \ldots x(12) x(7) \ldots x(12) x(13) \ldots x(18) x(13) \ldots x(18) \| \\
& x(19) \ldots x(24) x(19) \ldots x(24) x(25) \ldots x(30) x(25) \ldots x(30) x(31) \ldots x(36) x(31) \ldots x(36) \| \\
& x(19) \ldots x(24) x(19) \ldots x(24) x(25) \ldots x(30) x(25) \ldots x(30) x(31) \ldots x(36) x(31) \ldots x(36) \| \\
& x(37) \ldots x(42) x(37) \ldots x(42) x(43) \ldots x(48) x(43) \ldots x(48) x(49) \ldots x(54) x(49) \ldots x(54) \| \\
& x(37) \ldots x(42) x(37) \ldots x(42) x(43) \ldots x(48) x(43) \ldots x(48) x(49) \ldots x(54) x(49) \ldots x(54)
\end{aligned}
$$

Remark 2 : In the definition of $\operatorname{strcopy}(x, i, n)$, the term $x(1+t(i-1, n)) x(2+t(i-$ $1, n)) \ldots x(t(i, n))$ means the concatenation of all the elements between $x(1+t(i-1, n))$ and $x(t(i, n))$. In other words : $x(1+t(i-1, n)) x(2+t(i-1, n) \ldots x(t(i, n))=x(1+$ 
$t(i-1, n)) x(2+t(i-1, n) \ldots x(j) \ldots x(-1+t(i, n)) x(t(i, n))$ where $1+t(i-1, n) \leq$ $j \leq t(i, n)$.

Proof of Proposition 1 : It suffices to show that $3 D C T \leq_{m}^{P}$ Problem 5.

Let us suppose that the procedure Generalize solves Problem 5 and we want to show how to build a procedure Sol3DCT which solves 3DCT.

The procedure Sol3DCT takes as input a binary string $\mathrm{x}$, an integer $\mathrm{n}$ and returns as output the matrix $A$ of size $n$ such that $g_{2}(A)=x$. The procedure Generalize takes as input :

$-\mathrm{p}$ the dimension of the matrices

- two binary strings $x$ and $y$

- two matrices $V$ and $W$

and returns as output :

- two matrices $C$ and $D$ such that :

$-g_{2}(C . * V)=g_{2}(D . * V)=x$ and $g_{2}(C . * W)=g_{2}(D . * W)=y$

We show in the procedure below how to use Generalize as a subroutine to solve Sol3DCT.

Procedure Sol3DCT( $n$ : integer, $\mathbf{x}:$ string, var A : matrix );

$\mathrm{V}, \mathrm{W}, \mathrm{C}, \mathrm{D}:$ matrix

$\mathrm{p}, \mathrm{i}, \mathrm{j}, \mathrm{k}$ : integer

$\mathrm{z}$ : string

begin

1: $\quad V \longleftarrow$ MOnes $(2 n)$

2: $\quad W \longleftarrow$ Mones $(2 n)$

3: $\quad z \longleftarrow \operatorname{duplic}(x, n)$

4: $\quad p \longleftarrow 2 \times n$

5: $\quad$ Generalize $(p, z, z, V, W, C, D)$

6: $\quad$ For $\mathrm{i}=1$ to $\mathrm{n}$ do

7: $\quad$ For $\mathrm{j}=1$ to $\mathrm{n}$ do

8: $\quad$ For $\mathrm{k}=1$ to $\mathrm{n}$ do

9 :

10 :

$11:$

12 : 
The terms of the matrix $C$ are :

$$
\begin{cases}C(i, j, k)=A(i, j, k), & \text { if } 1 \leq i, j, k \leq n \\ C(i, j+n, k)=0, & \text { if } 1 \leq i, j, k \leq n \\ C(i+n, j, k)=0, & \text { if } 1 \leq i, j, k \leq n \\ C(i+n, j+n, k)=A(i, j, k), & \text { if } 1 \leq i, j, k \leq n \\ C(i, j, k+n)=0, & \text { if } 1 \leq i, j, k \leq n \\ C(i, j+n, k+n)=A(i, j, k), & \text { if } 1 \leq i, j, k \leq n \\ C(i+n, j, k+n)=A(i, j, k), & \text { if } 1 \leq i, j, k \leq n \\ C(i+n, j+n, k+n)=0, & \text { if } 1 \leq i, j, k \leq n\end{cases}
$$

The terms of the matrix $D$ are :

$$
\begin{cases}D(i, j, k)=0, & \text { if } 1 \leq i, j, k \leq n \\ D(i, j+n, k)=A(i, j, k), & \text { if } 1 \leq i, j, k \leq n \\ D(i+n, j, k)=A(i, j, k), & \text { if } 1 \leq i, j, k \leq n \\ D(i+n, j+n, k)=0, & \text { if } 1 \leq i, j, k \leq n \\ D(i, j, k+n)=A(i, j, k), & \text { if } 1 \leq i, j, k \leq n \\ D(i, j+n, k+n)=0, & \text { if } 1 \leq i, j, k \leq n \\ D(i+n, j, k+n)=0, & \text { if } 1 \leq i, j, k \leq n \\ D(i+n, j+n, k+n)=A(i, j, k), & \text { if } 1 \leq i, j, k \leq n\end{cases}
$$

The main idea of the design of the Collision-resistant hash function $H_{3}$ is that :

- the hash function $H_{3}$ is the composition of two functions $H_{1}$ and $H_{2}$,

- the function $H_{1}$ is a function for which Problem 1, Problem 2 and Problem 3 can't be solved efficiently and $H_{1}$ is not a compression function.

$-H_{2}$ is a hash function such as SHA-256, RIPEMD, or HAVAL, ....

Notation 1 Let us consider two vectors $V_{1}$ and $V_{2}$. We say that $V_{1}$ is not a linear combination of $V_{2}$ and we note $V_{1}$ is NLC of $V_{2}$ if and only if $\nexists \alpha \in \mathbb{R}$ such that $V_{1}=\alpha V_{2}$. 
Two matrices $F, G \in N_{+}^{n \times n \times n}$ verify the hypotheses (7) if and only if :

$$
F \neq G
$$

$\forall i, j$ such that $1 \leq i, j \leq n$, the vector $\mathrm{F}(\mathrm{i}, \mathrm{j}, *)$ is $\mathrm{NLC}$ of the vector $\mathrm{G}(\mathrm{i}, \mathrm{j}, *)$

$\forall j, k$ such that $1 \leq j, k \leq n$, the vector $\mathrm{F}(*, \mathrm{j}, \mathrm{k})$ is $\mathrm{NLC}$ of the vector $\mathrm{G}(*, \mathrm{j}, \mathrm{k})$

$\forall i, k$ such that $1 \leq i, k \leq n$, the vector $\mathrm{F}(\mathrm{i}, *, \mathrm{k})$ is $\mathrm{NLC}$ of the vector $\mathrm{G}(\mathrm{i}, *, \mathrm{k})$

$\forall i, j$ such that $1 \leq i, j \leq n$, the vector $\mathrm{F}(\mathrm{i}, \mathrm{j}, *)$ is $\mathrm{NLC}$ of the vector $\operatorname{VOnes}(\mathrm{n})$

$\forall j, k$ such that $1 \leq j, k \leq n$, the vector $\mathrm{F}(*, \mathrm{j}, \mathrm{k})$ is NLC of the vector VOnes(n)

$\forall i, k$ such that $1 \leq i, k \leq n$, the vector $\mathrm{F}(\mathrm{i}, *, \mathrm{k})$ is NLC of the vector VOnes(n)

$\forall i, j$ such that $1 \leq i, j \leq n$, the vector $\mathrm{G}(\mathrm{i}, \mathrm{j}, *)$ is NLC of the vector VOnes(n)

$\forall j, k$ such that $1 \leq j, k \leq n$, the vector $\mathrm{G}(*, \mathrm{j}, \mathrm{k})$ is NLC of the vector VOnes(n)

$\forall i, k$ such that $1 \leq i, k \leq n$, the vector $\mathrm{G}(\mathrm{i}, *, \mathrm{k})$ is $\mathrm{NLC}$ of the vector VOnes(n)

The matrices $V$ and $W$ used as entries in the procedures $H_{1}$ and $H_{3}$ below verify the hypotheses defined by Equations (7). We note $\epsilon$ the empty chain. Let us define the function VectMat which takes as input a vector $V e c t$ of size $n^{3}$ and returns as output an equivalent matrix $A$ of size $n \times n \times n$.

Procedure VectMat (Vect: Table[1..n $\left.n^{3}\right]$ of bit; Var A : Table[1..n, 1..n, 1..n] of bit ) Var $\mathrm{i}, \mathrm{j}, \mathrm{k}, \mathrm{t}$ : integer

Begin

$t \leftarrow 1$

For $\mathrm{i}=1$ to $\mathrm{n}$ do

For $\mathrm{j}=1$ to $\mathrm{n}$ do

For $\mathrm{k}=1$ to $\mathrm{n}$ do

$A(i, j, k) \leftarrow V e c t(t)$

$t \leftarrow t+1$

endfor

endfor

endfor

End

The function $H_{1}$ is defined as follows : 


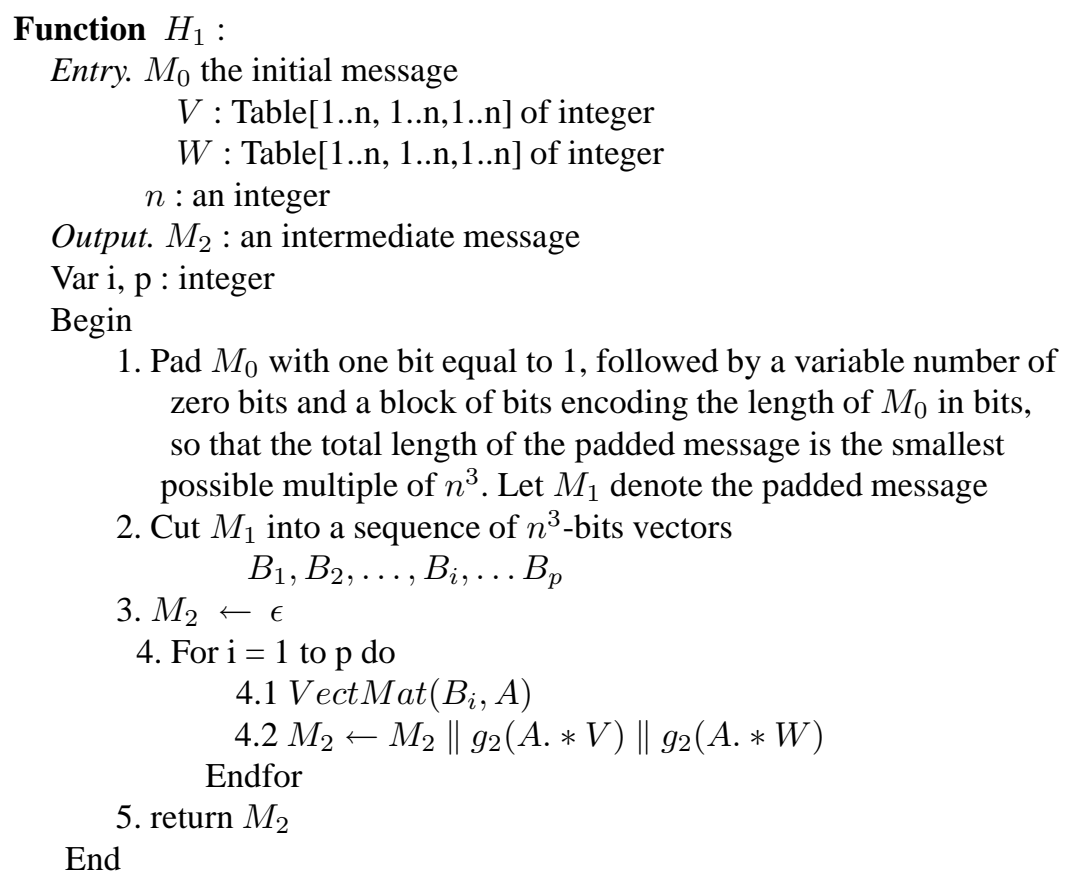

Our hash function $H_{3}$ is defined as the composition of the function $H_{1}$ and $H_{2}$, where $H_{2}$ is a hash function such as SHA-256, RIPEMD, HAVAL... The matrices $V$ and $W$ used as entry in the hash function $H_{3}$ must verify the hypotheses defined in Equations (7). To obtain the hash of the message $M_{0}$ by $H_{3}$, we proceed as follows :

- we obtain the intermediate message $M_{2}$ by application of the function $H_{1}$ to the message $M_{0}$,

- by application of the hash function $H_{2}$ to $M_{2}$, we build the hash of the initial message.

Formally, the hash function is defined as follows :

Procedure $\mathrm{H}_{3}$ :

Entry. $M_{0}$ the initial message

$$
\begin{aligned}
& V: \text { Table[1..n, 1..n, } 1 . . n] \text { of integer } \\
& W: \text { Table[1..n, 1..n,1..n] of integer } \\
& n: \text { an integer }
\end{aligned}
$$

Output. Result $:$ the hash of the message $M_{0}$

Begin

$$
\begin{aligned}
& M_{2} \leftarrow H_{1}\left(M_{0}, V, W, n\right) \\
& \text { Result } \leftarrow H_{2}\left(M_{2}\right)
\end{aligned}
$$

End

\section{Comment :}

We can represent roughly the function $H_{1}$ as follows : 


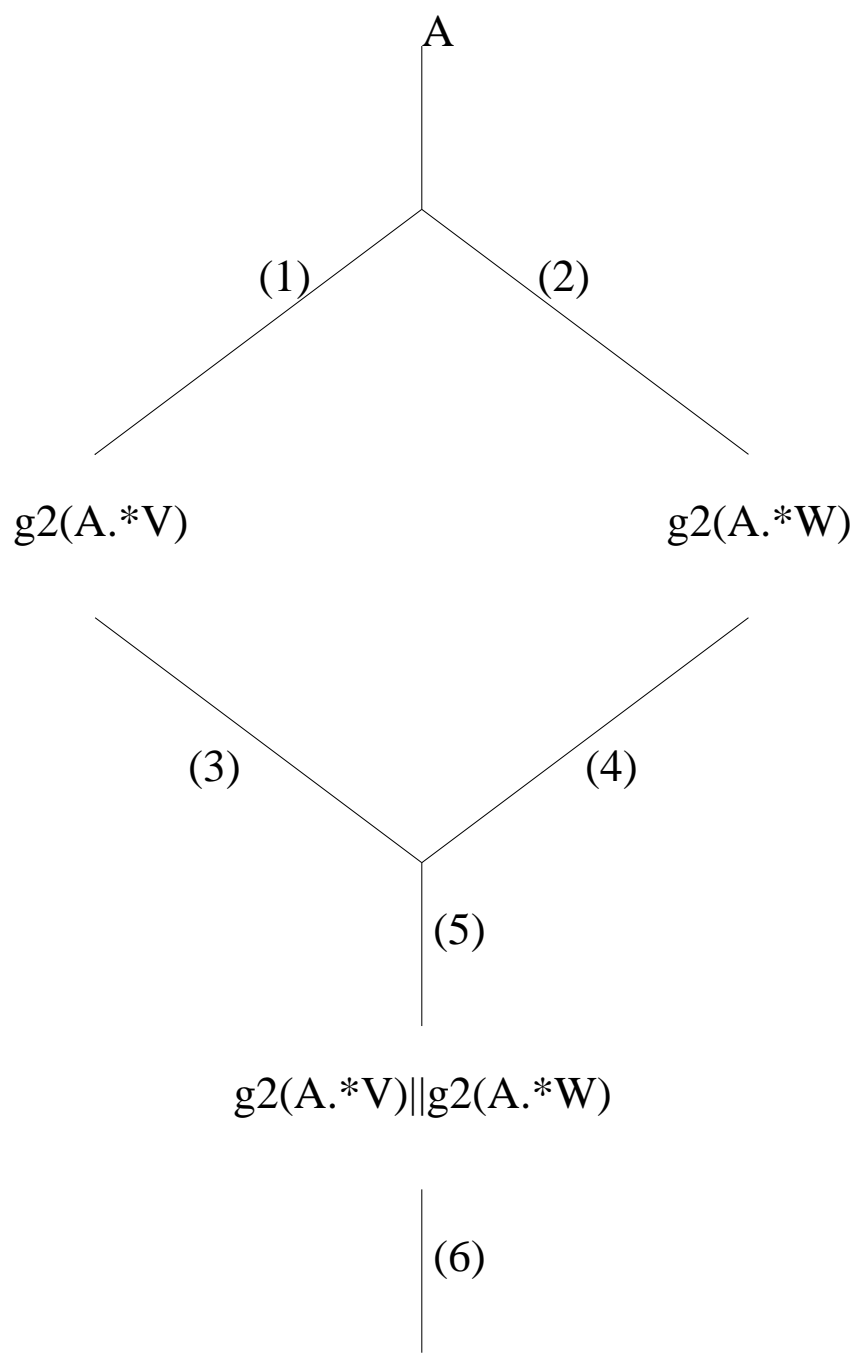

Figure 1. Roughly Representation of the function $H_{1}$

In the Figure 1 :

- the aim of the branches (1) and (2) is to make that the Problem 2 and Problem 3 are difficult to solve efficiently for the function $\mathrm{H}_{3}$

- the aim of the branch (6) is to make sure that Problem 1 is difficult to solve efficiently for the function $\mathrm{H}_{3}$

During some attacks, an adversary is needed to solve the following problem :

\section{Problem 6 :}

Instance : Matrices $\mathrm{A}, \mathrm{V}, \mathrm{W}$ 
Binary strings : $g_{2}(A . * V)$ and $g_{2}(A . * W)$

Query: Find a matrix $B$ such that $A \neq B$ and :

$$
\begin{aligned}
g_{2}(A * V) & =g_{2}(B * * V) \\
g_{2}(A * W) & =g_{2}(B * * W)
\end{aligned}
$$

Based on Problem 5, we deduce that Problem 6 is NP-Complete.

Second Preimage attack and Collision of the function $\mathrm{H}_{3}$ are difficult because :

- Problem 5 and Problem 6 are NP-Complete,

- From the fact that $V$ and $W$ verify the hypotheses (7), we deduce that if we take two matrices $A$ and $B$ such that $A \neq B$, then we would probably have $g_{2}\left(A\right.$ * *V) $\| g_{2}(A$ ** $W) \neq g_{2}(B . * V) \| g_{2}(B . * W)$.

First Preimage attack of the function $H_{3}$ is difficult because the 3DCT is NP-Complete. Truncated differential attack of $H_{1}$ is possible, but the differential attack of $H_{3}$ is difficult because 3DCT is NP-Complete and also Problem 5 is NP-Complete.

\section{Numerical Simulation}

Let's consider the two messages $\mathrm{x} 1$ and $\mathrm{x} 2$ :

$$
\begin{aligned}
x 1= & d 131 d d 02 c 5 e 6 e e c 4693 d 9 a 0698 a f f 95 c \\
& 2 f c a b 58712467 e a b 4004583 e b 8 f b 7 f 89 \\
& 55 a d 340609 f 4 b 30283 e 488832571415 a \\
& 085125 e 8 f 7 c d c 99 f d 91 d b d f 280373 c 5 b \\
& d 8823 e 3156348 f 5 b a e 6 d a c d 436 c 919 c 6 \\
& d d 53 e 2 b 487 d a 03 f d 02396306 d 248 c d a 0 \\
& e 99 f 33420 f 577 e e 8 c e 54 b 67080 a 80 d 1 e \\
& c 69821 b c b 6 a 8839396 f 9652 b 6 f f 72 a 70 \\
& \\
x 2= & 1131 d d 02 c 5 e 6 e e c 4693 d 9 a 0698 a f f 95 c \\
& 2 f c a b 50712467 e a b 4004583 e b 8 f b 7 f 89 \\
& 55 a d 340609 f 4 b 30283 e 4888325 f 1415 a \\
& 085125 e 8 f 7 c d c 99 f d 91 d b d 7280373 c 5 b \\
& d 8823 e 3156348 f 5 b a e 6 d a c d 436 c 919 c 6 \\
& d d 53 e 23487 d a 03 f d 02396306 d 248 c d a 0 \\
& e 99 f 33420 f 577 e e 8 c e 54 b 67080280 d 1 e \\
& c 69821 b c b 6 a 8839396 f 965 a b 6 f f 72 a 70
\end{aligned}
$$


We have MD5(x1)=MD5(x2)=EFE502F744768114B58C8523184841F3

after applying our hash function on these messages using $n=8, V[i][j][k]=i+8 j+64 k$, $W[i][j][k]=700-(j+8 * k+64 * i)$ for $1 \leq i \leq n$ we obtain :

$H_{3}(\mathrm{x} 1)=5 \mathrm{fe} 0 \mathrm{e} 56 \mathrm{f} 9 \mathrm{a} 4 \mathrm{ab} 66 \mathrm{a} 47 \mathrm{~d} 73 \mathrm{ce} 660 \mathrm{a} 2 \mathrm{c} 4 \mathrm{eb}$ and

$H_{3}(\mathrm{x} 2)=620 \mathrm{e} 2 \mathrm{f} 3 \mathrm{cfe} 0 \mathrm{afc} 403 \mathrm{c} 0 \mathrm{a} 8343173526 \mathrm{fc}$.

It follows that $M D 5(x 1)=M D 5(x 2)$ whereas $H_{3}(x 1) \neq H_{3}(x 2)$.

\section{Conclusion}

From a classical hash function $H_{2}$, we have built a new hash function $H_{3}$ from which First Preimage attack, Second Preimage attack and Collision attack are difficult to solve. Our new hash function is a composition of functions. The construction used the NPcompleteness of Three-dimensional contingency tables and the relaxation of the constraint that a hash function should also be a compression function. The complexity of our new hash function increases with regard to the complexity of classical hash functions.

\section{Bibliographie}

[1] R. A. Brualdi, Matrices of Zeros and Ones with Fixed Row and Column Sum Vectors, Linear Algebra and its Applications, 33, 1980, pp. 159-231.

[2] L. Cox, Suppression methodology and statistical disclosure control, J. Amer. Statist. Assoc., 75(1980), pp. 377-385.

[3] I. P. Fellegi, On the question of statistical confidentiallity, J. Amer. Statist. Assoc., 67, (1972), pp. 7-18.

[4] D. R. Fulkerson, An upper bound for the permanent of a fully indecomposable matrix, Pacific J. Math., 10, 1960 , pp. 831-836.

[5] D. Gale, A Theorem on flows in networks, Pacific J. Math., 7, 1957 , pp. 1073-1082.

[6] R. M. Haber, Minimal term rank of a class of (0,1)-matrices, Canad. J. Math., 15, 1963 , pp. 188-192.

[7] Hongbo Yu, Xiaoyun Wang, Multi-Collision Attack on the Compression Functions of MD4 and 3-Pass Haval, Lecture Notes in Computer Science, 4817 , Springer 2007, pp. 206-226.

[8] Hongbo Yu, Gaoli Wang, Guoyan Zhang, Xiaoyun Wang, The Second-Preimage Attack on MD4, Lecture Notes in Computer Science, 3810 , Springer 2005, pp. 1-12.

[9] M. -Y. Kao, D. Gusfield Hongbo Yu, Efficient detection and protection of information in cross tabulated tables : Linear invariant set, SIAM J. Disc. Math., 6 (1993), pp. 460-473.

[10] Xiaoyun Wang, Hongbo Yu, Yiqun Lisa Yin, Efficient Collision Search Attack on SHA-0, Lecture Notes in Computer Science, 3621 , Springer 2005, pp. 1-16.

[11] Xiaoyun Wang, Yiqun Lisa Yin, Hongbo Yu, Finding Collisions in the Full SHA-1, Lecture Notes in Computer Science, 3621, Springer 2005, pp. 17-36.

[12] Xiaoyun Wang, Xuejia Lai, Dengguo Feng, Hui Cheng, Xiuyuan Yu, Cryptanalysis of the Hash Functions MD4 and Ripemd, Lecture Notes in Computer Science, 3494 , Springer 2005, pp. 1-18.

[13] Xiaoyun Wang, Hongbo Yu, How to Break MD5 and Other Hash Functions, Lecture Notes in Computer Science, 3494 , Springer 2005, pp. 19-35. 
[14] Bert den Boer, Antoon Bosselaers, Collisions for the compression functions of MD5, Lecture Notes in Computer Science, 765, Springer 1994, pp. 293-304.

[15] R. W. Irving, M. R. Jerrum, Three-Dimensional Statistical Data Security Problems, SIAM J. Comput., Vol. 23, No 1, pp. 170-184, 1994.

[16] H. J. Ryser, Combinatorial properties of matrices of zeros and ones, Canad. J. Math., Vol. 9, pp. 371-377, 1957.

[17] G. Sande, Automated cell suppression to preserve confidentiality of business statistics, Statist. J. United Nations ECE 2 (1984) pp. 33-41. 